\title{
Building and Assessing a Constrained Clustering Hierarchical Algorithm
}

\author{
Eduardo R. Concepción Morales ${ }^{1}$ and Yosu Yurramendi Mendizabal ${ }^{2}$ \\ ${ }^{1}$ University of Cienfuegos, Faculty of Informatics, Cuatro Caminos, Cienfuegos, Cuba \\ econcep@ucf.edu.cu \\ ${ }^{2}$ University of the Basque Country/EHU, Campus Guipuzcoa, Department of Computer \\ Science and Artificial Intelligence, Donostia-San Sebastian, Spain \\ yosu.yurramendi@ehu.es
}

\begin{abstract}
Unsupervised classification or clustering has been used in many disciplines and contexts. Traditional methodologies are mostly based on the minimization of the distance between data and the cluster means without considering any other possible relationship present in data, e.g., spatial interactions. A constrained hierarchical agglomerative algorithm with an aggregation index is introduced which uses neighbouring relations present in the data. Experiments show the behaviour of the proposed constrained algorithm in different situations.
\end{abstract}

Keywords: constrained clustering methods, agglomerative hierarchical classification.

\section{Introduction}

Unsupervised classification or clustering has been used in many disciplines and contexts [1]. The goal of clustering is to partition a given set of data in homogeneous groups.

The notion of homogeneity has to be formalized in order to give a precise meaning to the results of clustering. Traditional methodologies are based on the minimization of distance between data and the cluster means without considering any other possible relationship present in data, e.g., spatial interactions [2].

Other approaches use separation measures, connectivity measures, compactness measures or mixed compactness/separation measures [3, 4]. In practice clustering is performed in many different frameworks, with different objectives, with more or less heuristic methods, and can be considered an experimental technique by nature.

One of the common applications of clustering is image segmentation. In image segmentation, particularly in region-based segmentation, pixels are classified in clusters based on their appearance while a hard constraint is imposed on cluster (spatial) connectivity. Here we consider the segmentation problem similar to the (constrained) clustering problem.

When considering spatially based applications objects are related in terms of space (contiguity) and feature values (compactness). For example, in the case of image 
segmentation, in general, nearby pixels have similar characteristics and therefore should belong to the same cluster. This fact is in correspondence with Tobler's first law of geography which states that everything is related to everything else, but near things are more related than distant things [5].

The idea of using neighborhood relations to model spatial interactions is the basis of various approaches such as Markov Random Fields [6]. It has also been used in the context of dimensionality reduction [7].

In this paper we propose a modified clustering hierarchical algorithm which uses an aggregation function based on pairwise intercluster distances. It takes into account spatial interactions which are modeled by means of a contiguity relation. At each step only those clusters are considered to be merged which are spatially contiguous. When calculating intercluster distances we use those members of the clusters which are across the border and are contiguous. We show that this aggregation does not lead to inversions.

Section 2 gives a description of the clustering model we assume. In section 3 some experimental results are presented which show the behaviour of the proposed constrained algorithm in different situations. Finally, section 4 presents conclusions and future work.

\section{Clustering Model}

Let us consider the data set $X=\left\{x_{1}, x_{2}, \ldots, x_{\mathrm{n}}\right\}$ which represents some abstract objects $o_{i}, i=1, \ldots, n$, where $x_{i}$ represents some scalar which describes the object $o_{i}$. In the following we will also use indices to denote objects, i.e., $i$ represents object $o_{i}$. A connection matrix $A=\left[a_{i j}\right]$ is used where $a_{i j}=1$ if objects $o_{i}$ and $o_{j}$ are contiguous or adjacent and $a_{i j}=0$ otherwise. The neighborhood of an object is the set of adjacent objects according to the connection matrix. In the case of image segmentation the contiguity relation can represent the 4- or 8- adjacency which is equivalent to a neighborhood of radius 1 and $\sqrt{ } 2$, respectively. We can also consider an arbitrary neighborhood of radius $r$ around any object.

Let $A_{i}$ be the number of neighbors of object $o_{i}$ and $A_{\text {Total }}$ the total number of neighbors, i.e,

$$
A_{\text {Total }}=\sum_{i=1}^{n} A_{i}=\sum_{i=1}^{n} \sum_{j=1}^{n} a_{i j}
$$

Assume we want to cluster these data into $K$ disjoint clusters, i.e., we want to obtain a partition $P=\left\{P_{1}, P_{2}, \ldots, P_{\mathrm{K}}\right\}$. Two clusters $P_{\mathrm{k}}$ and $P_{1}$ are said to be contiguous if at least one member of $P_{\mathrm{k}}$ is contiguous to at least one member of $P_{1}$. We will denote by $n_{k l}$ be the number of related pairs of objects between contiguous clusters $P_{\mathrm{k}}$ and $P_{\mathrm{l}}$, that is the number of links across the border of the two clusters. For a given cluster $P_{\mathrm{k}}$ the value $n_{k k}$ is the number of connections inside the cluster.

We will denote the weighted variance of the data set as

$$
s_{A}^{2}=\frac{1}{A_{\text {Total }}} \sum_{i=1}^{n} A_{i}\left(x_{i}-\bar{x}_{A}\right)^{2}
$$

where 


$$
\bar{x}_{A}=\frac{1}{A_{\text {Total }}} \sum_{i=1}^{n} A_{i} x_{i}
$$

represents the weighted mean of the data set. In both cases weights are defined according to the connection matrix.

The idea is that when obtaining theses values for a given region the contribution of each data point is proportional to the number of its neighbors. This way the data points located near the boundaries will contribute less to the cluster statistics. We claim this makes sense, for instance, in image segmentation applications.

In order to evaluate the quality of clustering several measures have been proposed [8]. Pairwise distances can define a global measure which favors certain properties such as low intra-cluster variability. This is the idea of the k-means algorithm $[9,10]$. It is well-known that the $k$-means cost function is equivalent to the average squared distance between all points [6]:

$$
\sum_{i=1}^{n} \sum_{j=1}^{n}\left(x_{i}-x_{j}\right)^{2}=2 n \sum_{i=1}^{n}\left(x_{i}-\bar{x}\right)^{2}=2 n^{2} s^{2}
$$

In some clustering problems the local data structure might be more relevant in order to identify clusters. Based on this fact different measures of the quality of clustering can be used. Here we propose a restricted version of the squared pairwise distances. Taking into account the contiguity relation between data points it can be shown that:

$$
\sum_{i=1}^{n} \sum_{j=1}^{n} a_{i j}\left(x_{i}-x_{j}\right)^{2}=2 A_{\text {Total }} s_{A}^{2}\left(1-I^{*}\right)=2 A_{\text {Total }} s_{A}^{2} C^{*}
$$

The values $I^{*}$ and $C^{*}$ are modified versions of the global Moran and Geary indices of spatial autocorrelation and defined as follows [11].

$$
C^{*}=\frac{\sum_{i=1}^{n} \sum_{j=1}^{n} a_{i j}\left(x_{i}-x_{j}\right)^{2}}{2 A_{\text {Total }} s_{A}^{2}}
$$

The reader is referred to [12,13] for the definitions of the global Moran and Geary indices of spatial autocorrelation.

This result resembles the one obtained in Eq. 3. The main difference is the factor $C^{*}=\left(1-I^{*}\right)$. The restricted pairwise squared distances are in fact related to the variance and spatial autocorrelation of data, taking into account the definitions given above. Moreover, if we consider the particular case when the neighborhood of each data point spans over the whole set of points, i.e., the data set is a clique, then we obtain the result of Eq. 3 .

For a given partition of cardinality $K$ we can write the following decomposition of the sum of restricted pairwise distances or the local variance $[14,15]$ :

$$
\begin{array}{r}
\frac{1}{A_{\text {Total }}} \sum_{i=1}^{n} \sum_{j=1}^{n} a_{i j}\left(x_{i}-x_{j}\right)^{2}=\sum_{k=1}^{K} \frac{n_{k k}}{A_{\text {Total }}} \frac{1}{n_{k k}} \sum_{i \in P_{k}} \sum_{j \in P_{k}} a_{i j}\left(x_{i}-x_{j}\right)^{2}+ \\
\sum_{k=1}^{K} \sum_{l=1}^{K} \frac{n_{k l}}{A_{\text {Total }}} \frac{1}{n_{k l}} \sum_{i \in P_{k}} \sum_{j \in P_{l}} a_{i j}\left(x_{i}-x_{j}\right)^{2}
\end{array}
$$


The first component accounts for the intra-cluster homogeneity and the second one quantifies the inter-cluster heterogeneity. The second component emphasizes the difference between two given clusters taking into consideration the connected members which are located across the boundaries.

In this context, as far as the local variance is constant for a given data set, minimizing inter-cluster homogeneity is equivalent to maximize inter-cluster heterogeneity.

We will define an aggregation based on the concept of heterogeneity of the clusters. More precisely, for any pair of neighboring clusters, the dissimilarity between them will be

$$
d\left(P_{k}, P_{l}\right)=\frac{1}{n_{k l}} \sum_{i \in P_{k}} \sum_{j \in P_{l}} a_{i j}\left(x_{i}-x_{j}\right)^{2}
$$

\subsection{The Algorithm}

With the idea of producing segmentations at various levels of resolution, an agglomerative hierarchical strategy will be used. The initial state of the algorithm is to assign every object to a cluster. The algorithm proceeds to select a pair of neighboring clusters with minimum dissimilarity and merge them. After this step, dissimilarities between the just created cluster and the rest of the adjacent clusters are updated.

In fact we are using a constrained version of the average link method [8]. The algorithm only takes into consideration neighboring cluster and neighboring members of candidate clusters. This is a hard constraint. A softer constraint can be defined which considers only neighboring clusters but uses all members to calculate the dissimilarity. We do not analyze this case here.

After merging any two clusters, it is necessary to update the dissimilarities with the rest; this is the reduction step. Two strategies are available: (1) use initial data, (2) use the dissimilarities of the previous step. This could be accomplished using the LanceWilliams-Jambu formula [16,17].

In this case we can show that the dissimilarities between a newly formed cluster and the rest can be obtained as

$$
d\left(P_{k} \cup P_{l}, P_{m}\right)=\frac{n_{k m} d\left(P_{k}, P_{m}\right)+n_{l m} d\left(P_{l}, P_{m}\right)}{n_{k m}+n_{l m}}
$$

The problem of inversions or reversals in the series of agglomerations does not occur here. It readily follows from the fact that in the right hand side of Eq. 7 we have a linear convex combination. The proof is omitted for reason of space.

As a result of the imposed constraint the number of needed comparisons can be significantly reduced. The running time is typically dominated by the time required for selecting the cluster pair to be merged. Efficient implementations can be obtained if we maintain a list of neighbouring clusters ordered by the dissimilarity for each cluster in the current set.

\section{Experiments}

In this section we show two types of experiments. First, a toy example is used to illustrate the behaviour of the constrained algorithm compared with the unconstrained or 


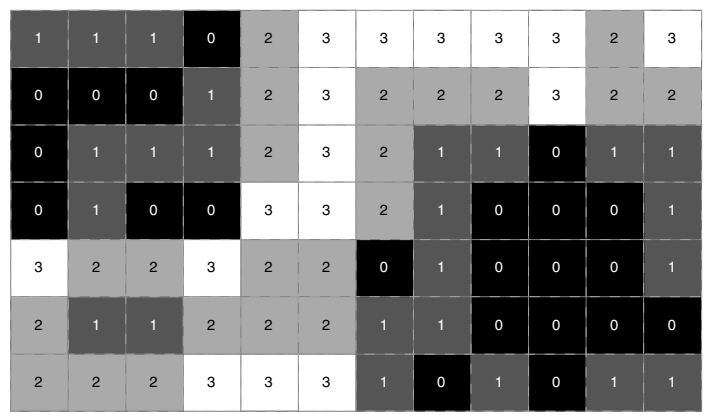

Fig. 1. Test image with four intensity values

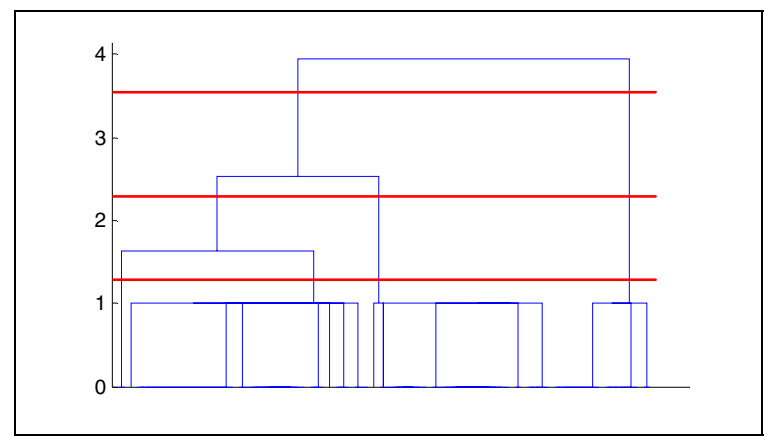

Fig. 2. Dendrogram obtained with the constrained algorithm using 8-adjacency

classical version. After that we use the algorithm to segment a region of interest in an MRI image. We compared the results with a manual segmentation provided by an expert.

In Fig. 1 we used a test image with four intensity values. We applied both the constrained algorithm and the classical average linkage method.

In Fig. 2 the resulting dendrogram using 8-adjacency is shown. Dendrogram is cut at different levels. The results in Fig. 3 corresponding to the constrained algorithm show that all partitions are composed by connected clusters.

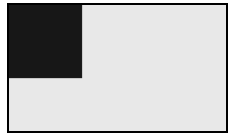

(a)

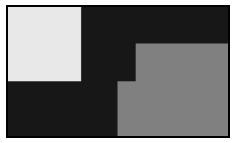

(b)

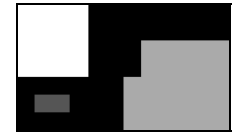

(c)

Fig. 3. Partitions obtained from dendrogram: (a) 2 clusters, (b) 3 clusters, (c) 4 clusters

An interesting result is obtained in Fig. 3 (c) with 4 connected clusters which describe a small region of 1's, 2 regions of 0's and 1's, and 1 region of 2's and 3's, while with the classical average linkage method the original image is obtained. 
Results of the constrained clustering algorithm depend on the contiguity relation defined over the data set. Instead of using common 4- or 8-adjacency, we can define neighborhoods of certain radius. For example, in Fig. 4 we use a checkerboard image with added Gaussian noise. Cases from (b) to (d) show that as neighborhood radius increases noise effect is reduced, and results are better from a perceptual point of view.

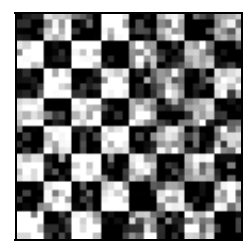

(a)

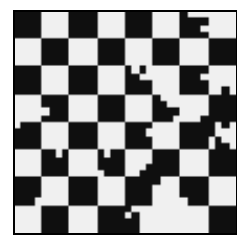

(b)

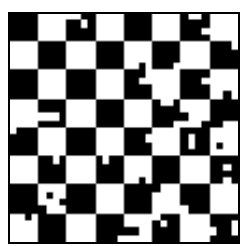

(c)

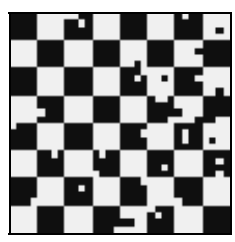

(d)

Fig. 4. (a) Checkerboard image with added Gaussian noise; (b) result with 8-adjacency; (c) result with neighborhood of radius 3 ; (d) result using neighborhood of radius 7

Finally, we used a small region of an MRI image together with the manual segmentation provided by an expert in Fig. 5 (a) and (b). In the manual segmentation 4 clusters are present: cerebrospinal fluid, white matter, gray matter, and non-brain tissue. In Fig. 5 (c) we show the partition with 4 clusters obtained with the classical average linkage method. Some differences can be detected, e.g., in the upper right corner where several different classes are identified by the average linkage method, while the expert classifies them as non-brain tissue.

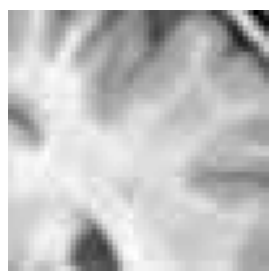

(a)

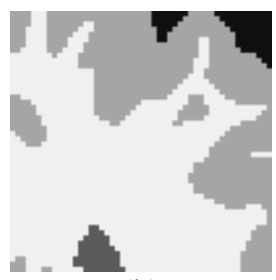

(b)

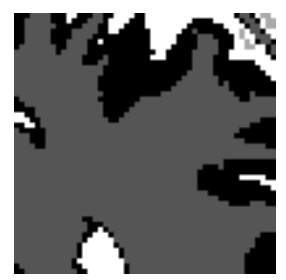

(c)

Fig. 5. (a) Small region of an MRI image, (b) its manual segmentation, and (c) partition with 4 clusters obtained with the classical average linkage method

We ran the constrained algorithm using 8-adjacency, and neighborhoods of radius 3 and 4. In Fig. 6 the obtained dendrogram using a neighborhood of radius 4 is shown. We empirically selected 15 clusters for visualization in all 3 cases. This partition shows an adequate level of detail.

As can be seen an increment in the radius of the neighborhood leads to more detailed partition. Here again more classes are identified in the upper right corner. In general the partition obtained in Fig. 7 (c) is in good correspondence, from a perceptual point of view, with the manual segmentation given by the expert. 


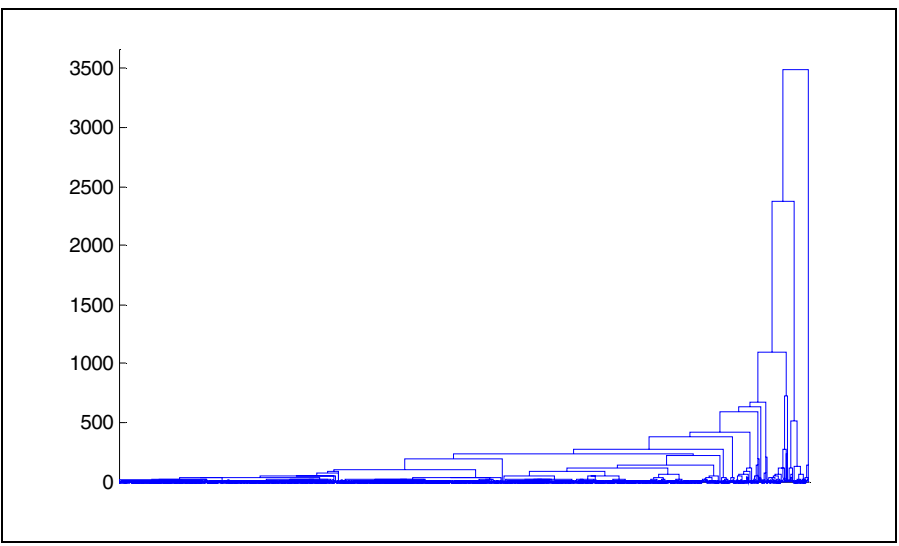

Fig. 6. Obtained dendrogram using a neighborhood of radius 4

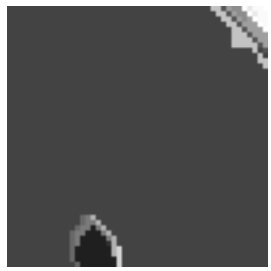

(a)

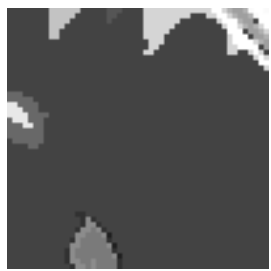

(b)

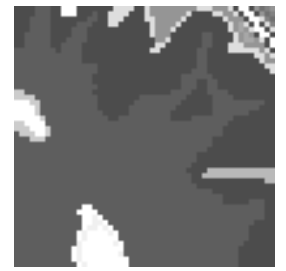

(c)

Fig. 7. (a) Results obtained with the constrained algorithm using 8-adjacency; and (b) neighborhoods of radius 3 , and (c) of radius 4

In all experiments big savings in running time were achieved with respect to the classical average linkage method.

\section{Conclusions and Future Work}

In this paper we have introduced a constrained hierarchical algorithm. The main constraint is about considering only neighbouring clusters as candidates to be merged. We define an aggregation index which takes into account neighbouring objects.

In the experiments we show the behaviour of the constrained algorithm in different situations. We compare results obtained with different neighbourhood relations, and for different partition sizes from a perceptual point of view. In comparisons we include the classical average linkage method as a reference. Results are in good correspondence with the expected partitions.

We are currently investigating the feasibility of different heuristics for automatically obtaining the number of clusters from the dendrogram.

The aggregation index takes into account the border zone of the candidate clusters. We also plan to include additional information about the potential boundary locations. This information could be obtained, e.g., applying an edge detector to the image. 


\section{References}

1. Jain, A.K., Murthy, M.N., Flynn, P.J.: Data Clustering: A Review. ACM Computing Surveys 31, 264-323 (1999)

2. Duda, R.O., Hart, P.E., Stork, D.G.: Pattern Classification. Wiley, New York (2000)

3. Puzicha, J., Hofmann, T., Buhmann, J.: A Theory of Proximity Based Clustering: Structure Detection by Optimization. Pattern Recognition 33, 617-634 (1999)

4. Shi, J., Malik, J.: Normalized Cuts and Image Segmentation. IEEE Trans. Patt. Anal. Mach. Intelligence 22, 888-905 (2000)

5. Tobler, W.R.: Cellular Geography. In: Gale, S., Olson, G. (eds.) Philosophy in Geography, pp. 379-386. Reidel, Dordrecht (1979)

6. Winkler, G.: Image Analysis, Random Fields and Markov Chain Monte Carlo Methods. Springer, Berlin (2003)

7. Roweis, S., Saul, L.: Nonlinear Dimensionality Reduction by Locally Linear Embedding. Science 290, 2323-2326 (2000)

8. Jain, A.K., Dubes, R.C.: Algorithms for Clustering Data. Prentice Hall, Englewoods Cliffs (1988)

9. Forgy, E.: Cluster Analysis of Multivariate Data: Efficiency Versus Interpretability of Classification. Biometrics 21, 768-780 (1965)

10. MacQueen, J.: Some Methods of Classification and Analysis of Multivariate Observations. In: Cam, L.M.L., Neyman, J. (eds.) Proceedings of Fifth Berkeley Symposium on Mathematical Statistics and Probability. University of California Press, Berkeley (1967)

11. Concepción, E., Yurramendi, Y.: Using Spatial Autocorrelation Measures for Data Clustering. In: 12th Conference of the Spanish Association for Artificial Intelligence, CAEPIATTIA, Salamanca (2007)

12. Cliff, A.D., Ord, K.J.: Spatial processes. Models and applications. Pion, London (1981)

13. Legendre, P., Legendre, L.: Numerical ecology. Elsevier, Amsterdam (1998)

14. Lebart, L.: Analyse Statistique de la Contiguïté. Publication de l'Institut de Statistiques de l'Université de Paris 28, 81-112 (1969)

15. Lebart, L.: Contiguity Analysis and Classification. In: Gaul, W., Opitz, O., Schader, M. (eds.) Data Analysis, pp. 233-244. Springer, Berlin (2000)

16. Jambu, M., Lebeaux, M.-O.: Cluster Analysis and Data Analysis. North-Holland, Amsterdam (1983)

17. Lance, G.N., Williams, W.T.: A General Theory of Classificatory Sorting Strategies. I. Hierarchical Systems. Computer Journal 9, 373-380 (1967) 